Article

\title{
The Usefulness of Quantitative Analysis of Blood-Brain Barrier Disruption Measured Using Contrast-Enhanced Magnetic Resonance Imaging to Predict Neurological Prognosis in Out-of-Hospital Cardiac Arrest Survivors: A Preliminary Study
}

\author{
Ho Il Kim ${ }^{1,+}$, In Ho Lee ${ }^{2,+}{ }^{(}$, Jung Soo Park ${ }^{1,3, *}$,, Da Mi Kim ${ }^{2}{ }^{(D}$, Yeonho You ${ }^{1}$, Jin Hong Min ${ }^{3}$, \\ Yong Chul Cho ${ }^{1}$, Won Joon Jeong ${ }^{1}$, Hong Joon Ahn ${ }^{1}$, Changshin Kang ${ }^{1}{ }^{\mathbb{D}}$ and \\ Byung Kook Lee ${ }^{4}$ (1) \\ 1 Department of Emergency Medicine, Chungnam National University Hospital, 282, Munhwa-ro, Jung-gu, \\ Daejeon 35015, Korea; laverhi@naver.com (H.I.K.); yyh1003@hanmail.net (Y.Y.); boxter73@naver.com (Y.C.C.); \\ gardenjun@hanmail.net (W.J.J.); jooniahn@hanmail.net (H.J.A.); changsiny@naver.com (C.K.) \\ 2 Department of Radiology, College of Medicine, Chungnam National University, 266, Munhwa-ro, Jung-gu, \\ Daejeon 35015, Korea; leeinho1974@hanmail.net (I.H.L.); damirad@cnuh.co.kr (D.M.K.) \\ 3 Department of Emergency Medicine, College of medicine, Chungnam National University, \\ Daejeon 35015, Korea; shiphid@hanmail.net \\ 4 Department of Emergency Medicine, Chonnam National University Medical School, Gwangju 61469, Korea; \\ bbukkuk@hanmail.net \\ * Correspondence: cpcr@cnu.ac.kr; Tel.: +82-42-280-6002 \\ + Ho Il Kim and In Ho Lee contributed equally to this work.
}

Received: 31 July 2020; Accepted: 16 September 2020; Published: 18 September 2020

\begin{abstract}
We aimed to evaluate neurological outcomes associated with blood-brain barrier (BBB) disruption using contrast-enhanced magnetic resonance imaging (CE-MRI) in out-of-hospital cardiac arrest (OHCA) survivors. This retrospective observational study involved OHCA survivors who had undergone CE-MRI for prognostication. Qualitative and quantitative analyses were performed using the presence of BBB disruption (pBD) and the BBB disruption score (sBD) in CE-MRI scans, respectively. For the sBD, 1 point was assigned for each area of $\mathrm{BBB}$ disruption, and 6 points were assigned when an absence of intracranial blood flow due to severe brain oedema was confirmed. The primary outcome was poor neurological outcome at 3 months (defined as cerebral performance categories 3-5). We analysed 46 CE-MRI brain scans (27 patients). Of these, 15 (55.6\%) patients had poor neurological outcomes. Poor neurological outcome group patients showed a significantly higher proportion of $\mathrm{pBD}$ than those in the good neurological outcome group $(22(88 \%)$ vs. $6(28.6 \%)$ patients, respectively, $p<0.001)$ and a higher sBD $(5.0(4.0-5.0)$ vs. $0.0(0.0-1.0)$ patients, $p<0.001)$. Poor neurological outcome predictions showed that the $\mathrm{sBD}$ had a significantly better prognostic performance (area under the curve (AUC) $0.95,95 \%$ confidence interval (CI) $0.84-0.99$ ) than the pBD (AUC $0.80,95 \%$ CI $0.65-0.90$ ). The sBD cut-off value was $>1$ point (sensitivity, $96.0 \%$; specificity, $81.0 \%$ ). The sBD is a highly predictive and sensitive marker of 3-month poor neurological outcome in OHCA survivors. Multicentre prospective studies are required to determine the generalisability of these results.
\end{abstract}

Keywords: heart arrest; prognosis; magnetic resonance imaging 


\section{Introduction}

Cardiac arrest (CA) is a common cause of death and disability [1]. Among more than 300,000 CA events that occur each year in the United States, the rate of survival to hospital discharge from out-of-hospital cardiac arrest (OHCA) has been reported to be $<10 \%$ [2]. Even when return of spontaneous circulation (ROSC) is achieved, approximately $30 \%$ of survivors have permanent brain damage [3]. Several prognostic methods have been suggested for predicting neurologic outcome. All prognostic tools are recommended $48-72 \mathrm{~h}$ after CA except for brain computed tomography (CT) and status myoclonus (within $24 \mathrm{~h}$ ) [4]. However, sedatives and neuromuscular blocking agent might result in misleading outcome predictions regarding the presence of myoclonus. Current prognostication guidelines suggest performing brain magnetic resonance imaging (MRI) 2-5 days after ROSC [5]. However, recent evidence has shown that MRI is better than brain $\mathrm{CT}$ at predicting neurological outcome within $3 \mathrm{~h}$ after ROSC [6-8].

Brain injury following cardiopulmonary resuscitation (CPR) is a significant cause of morbidity in survivors [9]. One of the most serious complications following CA is brain oedema, which is associated with poor neurological outcome and death [3]. Following cerebral ischemia-reperfusion, pathological alterations to the blood-brain barrier (BBB) play a decisive role in the ensuing formation of oedema [10]. As a result, BBB disruption is a major factor leading to permanent cerebral oedema after CA and resuscitation [11]. However, the relationship between BBB disruption after CA and neurological prognosis is rarely discussed in clinical studies, in contrast to animal studies. Our previous study is the first clinical study wherein we report that severe BBB disruption onset timing and severe BBB disruption are strongly associated with poor neurological outcomes; however, we did not observe a relationship between neurological outcome and the degree of BBB disruption [9]. Therefore, determining the degree of BBB disruption is likely to be very helpful in predicting the prognosis of patients with CA. Many studies have been conducted on BBB disruption.

The cerebrospinal fluid (CSF)-serum albumin quotient $\left(\mathrm{Q}_{\mathrm{A}}\right)$, calculated using CSF/serum-albumin is a gold standard numerical indicator used to determine the functional assessment of BBB disruption [12]. Contrast-enhanced magnetic resonance imaging (CE-MRI) has also been confirmed as a useful imaging test to determine the degree of BBB disruption, and this indicator can be visually confirmed [13].

To date, no study has reported the use of CE-MRI to measure BBB disruption as a prognostic factor for patients with $C A$. In addition, the relationship between the $Q_{A}$ and the degree of $B B B$ disruption measured using CE-MRI in patients with CA has not been identified. Therefore, we aimed to evaluate the usefulness of a quantitative analysis of BBB disruption measured using CE-MRI to predict neurological prognosis in OHCA survivors.

\section{Patients and Methods}

\subsection{Study Design and Patients}

In this retrospective observational study, we used prospectively collected data derived from adult comatose OHCA survivors treated with target temperature management (TTM) at Chungnam National University Hospital in Daejeon, Korea, between April 2019 and February 2020. The Institutional Review Board of Chungnam National University Hospital approved this study (CNUH-2020-06-022).

Our inclusion criteria comprised adult OHCA survivors (age, $\geq 18$ years) who were unconscious (Glasgow Coma Scale score, $\leq 8$ ) after ROSC and who had been treated with TTM. Exclusion criteria comprised patients: (1) aged $<18$ years; (2) with traumatic CA; (3) with an interrupted TTM (because of transfer from another facility or due to hemodynamic instability $(<60 \mathrm{mmHg}$ mean arterial pressure or $<90 \mathrm{mmHg}$ systolic blood pressure despite $\geq 6 \mathrm{~h}$ of the vasopressor support); (4) not eligible for TTM (i.e., because of intracranial haemorrhage, active bleeding, a known terminal illness, or a poor pre-CA neurological status), and; (5) who had been administered extracorporeal membrane oxygenation (ECMO). 


\subsection{Target Temperature Management Protocol}

The patients had been managed according to our previously published TTM protocol [14]. A target temperature was maintained at $33{ }^{\circ} \mathrm{C}$ for $24 \mathrm{~h}$ using feedback-controlled surface-cooling devices (Artic Sun ${ }^{\circledR}$ Energy Transfer Pads ${ }^{\mathrm{TM}}$; Medivance Corp, Louisville, CO, USA). Midazolam $(0.05 \mathrm{mg} / \mathrm{kg}$ intravenous bolus, followed by a titrated intravenous continuous infusion of $0.05-0.2 \mathrm{mg} / \mathrm{kg} / \mathrm{h}$ ) and cisatracurium $(0.15 \mathrm{mg} / \mathrm{kg}$ intravenous bolus, followed by an infusion of up to $0.3 \mathrm{mg} / \mathrm{kg} / \mathrm{h}$ ) were administered for sedation and to control shivering. Electroencephalography was performed if there was a persistent deterioration in a patient's level of consciousness, involuntary movements, or seizure. If there was evidence of electrographic seizure or a clinical diagnosis of seizure, an anti-epileptic drug was administered, namely, levetiracetam (loading dose $2 \mathrm{~g}$ bolus intravenously and maintenance dose, $1 \mathrm{~g}$ bolus twice daily, intravenously). All patients were treated with standard intensive care according to our institutional intensive care unit protocol.

\subsection{Data Collection and Primary Outcome}

The following data were collected from the database: age, sex, presence of a witness at the time of collapse, bystander CPR, first monitored rhythm, aetiology of CA, time from collapse to CPR (no flow time), time from CPR to ROSC (low flow time), time from ROSC to first and second MRI scan, and neurological outcome after CA.

The primary endpoint of this study was neurological outcome 3 months after CA. We measured neurological outcome 3 months after ROSC using the Glasgow Pittsburgh cerebral performance category (CPC) scale, either via face-to-face interviews or structured telephone interviews. Phone interviews were conducted by an emergency physician who had been fully informed of the protocol and was blinded to patient prognoses, the CE-MRI findings, and the $\mathrm{Q}_{\mathrm{A}}$. A poor neurological outcome was defined as a CPC score of 3,4 , or 5 .

\section{4. $Q_{A}$ measurement}

CSF was obtained via lumbar catheter drainage, and serum was collected through venepuncture. A lumbar catheter insertion was performed using a Hermetic ${ }^{\mathrm{TM}}$ lumbar catheter accessory kit (Integra Neurosciences, Plainsboro, NJ, USA), with the patient lying in a lateral decubitus position with hips and knees flexed. CSF albumin and serum albumin samples were both obtained at the same time within $6 \mathrm{~h}$ after ROSC and between $72 \mathrm{~h}$ and $96 \mathrm{~h}$ after ROSC. Albumin analysis was performed using a TBA-2000FR (Canon Medical Systems Corporation, Otawara, Japan). The $\mathrm{Q}_{\mathrm{A}}$ was calculated using the following formula: [albumin $\left.\mathrm{CSF}_{\mathrm{F}}\right] /\left[\right.$ albumin $\left._{\text {serum }}\right]$. The degree of BBB disruption was defined as follows: $Q_{A}>0.007$ (more than mild), $>0.01$ (more than moderate), and $\geq 0.02$ (severe).

\subsection{Qualitative and Quantitative Analyses of BBB Disruption Using CE-MRI}

In this study, we undertook qualitative and quantitative analyses using the presence of $B B B$ disruption (pBD) and the BBB disruption score (sBD) in CE-MRI scans, respectively. Our institution has a standardised CE-MRI protocol for nontraumatic OHCA survivors. If a patient's condition was haemodynamically stable and the patient's family consented to an MRI scan, all OHCA survivors underwent two MRI scans. The first MRI was obtained within $6 \mathrm{~h}$ after ROSC, and the second was obtained between $72 \mathrm{~h}$ and $96 \mathrm{~h}$ after ROSC. CE-MRI brain images were obtained using a 3T scanner (Achieva 3T, Philips Medical System, Andover, Netherlands). The $\mathrm{Q}_{\mathrm{A}}$ measurement and the CE-MRI brain images were both obtained simultaneously. The protocol for using the 3T scanner included a precontrast fluid attenuated inversion recovery (FLAIR) (TR/TE, 11,000/125 ms; section thickness, $5 \mathrm{~mm}$; section gap, $1.5 \mathrm{~mm}$; FOV, $220 \times 220 \mathrm{~mm}$; matrix, $316 \times 184$, number of slices, 24, and; number of excitations, 2) and a post-contrast FLAIR (TR/TE, 11,000/110 ms; section thickness, $5 \mathrm{~mm}$; section gap, $1.5 \mathrm{~mm}$; FOV, $220 \times 220 \mathrm{~mm}$; matrix, $316 \times 184$, number of slices, 24, and; number of excitations, 2) after the administration of gadobutrol (Gadovist, Bayer Healthcare, Berlin, Germany) at a dose of 
$0.1 \mathrm{mmol} / \mathrm{kg}$ (or up to a total dose of $10 \mathrm{~mL}$ ) and an injection rate of $0.5-1 \mathrm{~mL} / \mathrm{sec}$. The affected regions of the brain were selected by comparing each image using a slice-wise method.

The obtained CE-MRI brain images were interpreted by two board-certified neuroradiologists who were blinded to patient information. Gadobutrol was used as a contrast agent during the CE-MRI examination. The pBD on CE-MRI was identified through confirming gadobutrol leakage on postcontrast FLAIR scans compared to precontrast FLAIR scans. The $\mathrm{pBD}$ was evaluated according to brain regions (frontal, parietal, temporal, occipital, and cerebellum). One point was assigned for each area where BBB disruption was present, and 6 points were assigned when the absence of intracranial blood flow due to severe brain oedema was confirmed. Depending on the scoring system, a possible score could range from 0 to 6 points (Figure 1).
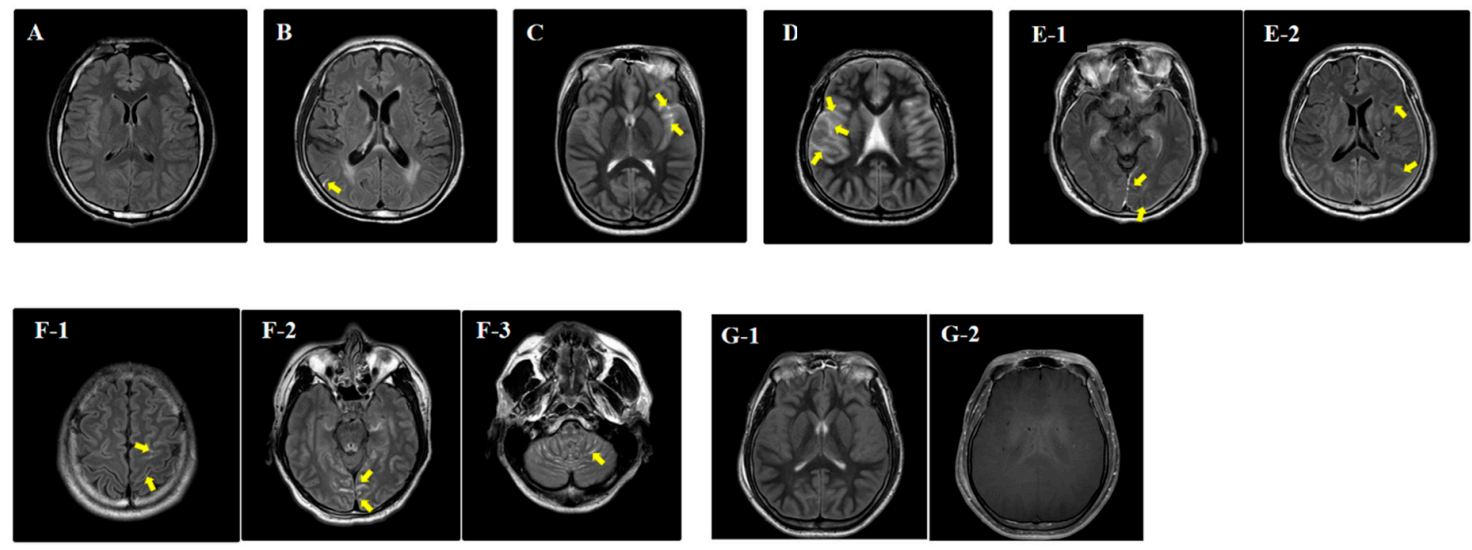

Figure 1. The blood-brain barrier disruption score using a post-contrast fluid-attenuated inversion recovery image. (A) Leptomeningeal enhancement in the subarachnoid space is not visible; a score of 0 . (B) Leptomeningeal enhancement in the right parietal sulcus is visible; a score of 1. (C) Leptomeningeal enhancement in the left sylvian fissure (lateral sulcus separating the frontal lobe from the temporal lobe) is visible; a score of 2. (D) Leptomeningeal enhancement in the right frontal, parietal and temporal sulci is visible; a score of 3. (E-1) Leptomeningeal enhancement in the left temporo-occipital sulci and, (E-2) left fronto-parietal sulci is visible; a score of 4. (F-1) Leptomeningeal enhancement in the left fronto-parietal sulci, (F-2) left temporo-occipital sulci and, (F-3) cerebellar folia is visible; a score of 5. (G-1) Leptomeningeal enhancement is not visible in the subarachnoid space and, (G-2) the intracranial blood flow is absent in the contrast enhanced T1 weighted MR; a score of 6 .

\subsection{Using CE-MRI to Analyse the Relationship between $Q_{A}$ and BBB Disruption}

We undertook a receiver operating characteristic (ROC) curve analysis to determine the performance of the $\mathrm{Q}_{\mathrm{A}}$ in predicting $\mathrm{BBB}$ disruption using CE-MRI. BBB disruption was assessed using the $\mathrm{sBD}$, which was redefined as binary. If the sBD was equal to or exceeded the cut-off value of the highest area under the ROC (AUROC) capacity predicting poor neurological outcome at 3 months, this was classified as BBB disruption.

\subsection{Statistical Analysis}

Categorical variables are presented as frequencies and percentages, and continuous variables are presented as means and standard deviations, or as median and interquartile ranges, depending on the normality of the distribution. The normal distribution of data was analysed using a Shapiro-Wilk test. We compared categorical variables between the groups using Pearson's chi-squared- or Fisher's exact tests, as appropriate. We compared continuous variables between the groups using independent $\mathrm{t}$ - or Mann-Whitney U tests, as appropriate. AUROC analysis was performed to examine the relationship between the $\mathrm{Q}_{\mathrm{A}}$ and $\mathrm{BBB}$ disruption using CE-MRI and the prognostic performance of the $\mathrm{Q}_{\mathrm{A}}, \mathrm{pBD}$, and SBD for poor neurological outcome at 3 months after ROSC. The optimum cut-off values were determined using Youden's index (sensitivity + specificity -1$)$. Subsequently, we used a Delong test 
to determine differences in the relationship and prognostic performance. Data were analysed using SPSS for Windows, version 21 (IBM Corp., NY, NY, USA). ROC curves were calculated using MedCalc version 15.2.2 (MedCalc Software, Mariakerke, Belgium). A two-sided significance level of 0.05 was used to indicate statistical significance.

\section{Results}

\subsection{Patient Demographics}

During the study period, of 39 patients with OHCA who had been admitted to the intensive care unit for TTM, 1 had not undergone a first or a second MRI, 1 had undergone an MRI within $>6 \mathrm{~h}$ (first scan), 1 had undergone ECMO treatment, and a different scanning protocol (such as a diffusion-weighted image (DWI)-MRI only) had been used for 9 patients. As a result, 27 patients were enrolled in the study, of whom $19(70.4 \%)$ had undergone two CE-MRI scans.

Table 1 shows the baseline and CA characteristics according to neurological outcome. At 3 months after ROSC, $15(55.6 \%)$ patients had a poor neurological outcome. The median age was $60.0(40.0-70.0)$ years, and 21 patients $(77.8 \%)$ were men. The first brain MRI scans were obtained at a median of 2.6 (1.9-3.9) $\mathrm{h}$ after ROSC and the second brain MRI scans were acquired at a median of 76.7 (75.2-76.7) $\mathrm{h}$ after ROSC. Patients with poor neurological outcomes were less likely to have had a witnessed CA, bystander CPR, and shockable rhythms. Moreover, they had longer low flow and no flow times. Table 2 shows the individual values for $\mathrm{Q}_{\mathrm{A}}, \mathrm{pBD}$ and sBD for a total 27 patients.

Table 1. Baseline demographics and clinical characteristics of 27 patients.

\begin{tabular}{|c|c|c|c|c|}
\hline Characteristics & Cohort $(n=27)$ & Good Outcome $(n=12)$ & Poor Outcome $(n=15)$ & $p$-Value \\
\hline Age, years, median (IQR) & $60.0(40.0-70.0)$ & $60.5(46.5-69.5)$ & $60(40.0-74.0)$ & 0.764 \\
\hline Sex, male, $n(\%)$ & $21(77.8)$ & $11(91.7)$ & $10(66.7)$ & 0.182 \\
\hline \multicolumn{5}{|l|}{ Arrest characteristics } \\
\hline Witness arrest, $n(\%)$ & $18(66.7)$ & $11(91.7)$ & $7(46.7)$ & 0.014 \\
\hline Bystander CPR, $n(\%)$ & $17(63.0)$ & $11(91.7)$ & $6(40.0)$ & 0.006 \\
\hline Shockable rhythm, $n(\%)$ & $4(14.8)$ & $4(33.3)$ & $0(0.0)$ & 0.015 \\
\hline Cardiac aetiology, $n(\%)$ & $11(40.7)$ & $7(58.3)$ & $4(26.7)$ & 0.096 \\
\hline No flow time, $\min (\mathrm{IQR})$ & $3.5(0.0-25.5)$ & $0(0.0-2.5)$ & $21(7.8-36.3)$ & 0.005 \\
\hline Low flow time, $\min$ (IQR) & $23.0(9.0-31.0)$ & $15(6.5-23.5)$ & $30(20.0-39.0)$ & 0.017 \\
\hline ROSC to first MRI time, hr (IQR) & $2.62(1.87-3.86), 22$ * & $2.15(1.63-3.44), 10$ * & $2.75(2.21-5.41), 12$ * & 0.129 \\
\hline ROSC to second MRI time, hr (IQR) & $76.72(75.19-76.72), 24 *$ & $75.67(74.47-77.87), 11$ * & 77.18 (75.97-81.37), 13 * & 0.111 \\
\hline
\end{tabular}

IQR, interquartile range; CPR, cardiopulmonary resusciation; ROSC, return of spontaneous circulation; MRI, magnetic resonance imaging; ${ }^{*}$ Number of MRI scan in the analysis.

Table 2. Individual values for $\mathrm{Q}_{\mathrm{A}}, \mathrm{pBD}$ and $\mathrm{sBD}$ for a total of 27 patients.

\begin{tabular}{ccccccc}
\hline \multirow{2}{*}{ Case Number } & \multicolumn{2}{c}{$\mathbf{Q}_{\mathbf{A}}$} & \multicolumn{2}{c}{ pBD } & \multicolumn{2}{c}{ sBD } \\
\cline { 2 - 7 } & First & Second & First & Second & First & Second \\
\hline Patient 1 & 0.0132 & 0.0161 & Absence & Presence & 0 & 4 \\
Patient 2 & 0.0094 & & Absence & Absence & 0 & 0 \\
Patient 3 & 0.0083 & 0.0091 & Presence & Presence & 1 & 1 \\
Patient 4 & & 0.0065 & & Presence & & 4 \\
Patient 5 & & & Presence & Absence & 5 & 6 \\
Patient 6 & \multirow{2}{*}{0.0176} & Absence & Presence & 0 & 4 \\
Patient 7 & 0.0054 & & Absence & & 0 & \\
Patient 8 & 0.0083 & 0.0593 & Presence & Presence & 5 & 5 \\
Patient 9 & 0.0345 & & Presence & Presence & 4 & 4 \\
Patient 10 & 0.0097 & & Presence & Absence & 4 & 6 \\
Patient 11 & 0.0500 & 0.1188 & Presence & Presence & 2 & 5 \\
Patient 12 & 0.0100 & 0.0133 & Absence & Absence & 0 & 0 \\
\hline
\end{tabular}


Table 2. Cont.

\begin{tabular}{|c|c|c|c|c|c|c|}
\hline \multirow{2}{*}{ Case Number } & \multicolumn{2}{|c|}{$\mathbf{Q}_{\mathrm{A}}$} & \multicolumn{2}{|c|}{$\mathrm{pBD}$} & \multicolumn{2}{|c|}{ sBD } \\
\hline & First & Second & First & Second & First & Second \\
\hline Patient 13 & & & Presence & Presence & 4 & 3 \\
\hline Patient 14 & 0.0242 & 0.1000 & Presence & Presence & 5 & 5 \\
\hline Patient 15 & 0.0091 & 0.0057 & Absence & Absence & 0 & 0 \\
\hline Patient 16 & 0.0050 & 0.0061 & Absence & Absence & 0 & 0 \\
\hline Patient 17 & & 0.0049 & & Absence & & 0 \\
\hline Patient 18 & & 0.0071 & & Presence & & 4 \\
\hline Patient 19 & 0.0161 & 0.0615 & Presence & Presence & 5 & 5 \\
\hline Patient 20 & 0.0083 & 0.0029 & Presence & Absence & 3 & 0 \\
\hline Patient 21 & 0.0103 & & Presence & & 5 & \\
\hline Patient 22 & & & Absence & Presence & 0 & 2 \\
\hline Patient 23 & 0.0056 & 0.0200 & Presence & Presence & 5 & 5 \\
\hline Patient 24 & 0.0167 & & Presence & & 5 & \\
\hline Patient 25 & & 0.0621 & & Presence & & 2 \\
\hline Patient 26 & & 0.0250 & & Presence & & 5 \\
\hline Patient 27 & 0.0053 & 0.0029 & Absence & Absence & 0 & 0 \\
\hline
\end{tabular}

First: MRI or $\mathrm{Q}_{\mathrm{A}}$ was obtained within $6 \mathrm{~h}$ after ROSC; Second: MRI or $\mathrm{Q}_{\mathrm{A}}$ was obtained between $72 \mathrm{~h}$ and $96 \mathrm{~h}$ after ROSC. $\mathrm{Q}_{\mathrm{A}}$, cerebrospinal fluid-serum albumin quotient; $\mathrm{pBD}$, presence of blood-brain barrier disruption; $\mathrm{sBD}$, blood-brain barrier disruption score

\subsection{A Comparison of $p B D$ and $s B D$ Using $C E-M R I$ to Predict Neurological Outcome}

A total of 46 CE-MRI scans were acquired in 27 patients. Of these CE-MRI scans, 21 (45.7\%) showed a good neurological outcome and $25(55.3 \%)$ showed a poor neurological outcome. The locations of BBB disruption and each score for the 46 postcontrast FLAIR scans according to the BBB disruption scoring system are summarised in Table 3 . In total, 16 scans had scores of 0 , and 13 scans had scores of 5 . The poor neurological outcome group showed a significantly higher proportion of $\mathrm{pBD}$ and higher sBD scores than the good neurological outcome group (22 (88\%) vs. $6(28.6 \%)$ patients, $p<0.001$ and $5.0(4.0-5.0)$ vs. $0.0(0.0-1.0), p<0.001$; respectively; Figure 2$)$. A significantly higher AUROC capacity to predict poor neurological outcome at 3 months was found for sBD than for pBD $(0.95(95 \% \mathrm{CI}$ $0.84-0.99)$ vs. $0.80(95 \%$ CI $0.65-0.90), p=0.015)$, respectively (Figure 3$)$. The cut-off value of the sBD was $>1$ point, with sensitivity and specificity of $96.0 \%$ and $81.0 \%$, respectively (Table 4 ).

Table 3. Location and numbers of BBB disruption, scored according to BBB disruption scoring system in 46 CE-MRI scans.

\begin{tabular}{ccc}
\hline Score & Number & Location of BBB Disruption \\
\hline 0 & 16 & None \\
\hline 1 & 2 & Parietal lobe, $n=2$ \\
\hline 2 & 3 & $\begin{array}{c}\text { Frontal lobe + Parietal lobe, } n=1 \\
\text { Temporal lobe + Occipital lobe, } n=2\end{array}$ \\
\hline 3 & 2 & $\begin{array}{c}\text { Frontal lobe + Parietal lobe + Temporal lobe, } n=1 \\
\text { Parietal lobe + Temporal lobe + Occipital lobe, } n=1\end{array}$ \\
\hline 4 & 8 & Frontal lobe + Parietal lobe + Temporal lobe + Occipital lobe, $n=8$ \\
\hline 5 & 13 & Frontal lobe + Parietal lobe + Temporal lobe + Occipital lobe + Cerebellum, $n=13$ \\
\hline 6 & 2 & No internal carotid artery flow, $n=2$ \\
\hline
\end{tabular}


A. Presence of blood-brain barrier disruption

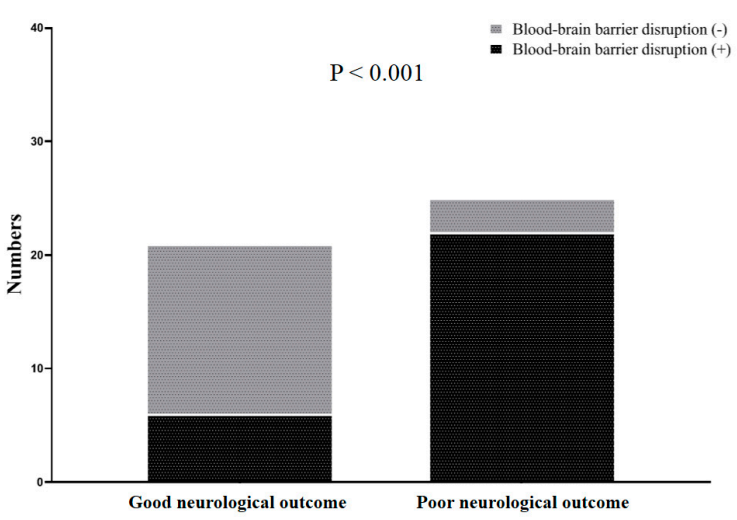

B. Blood-brain barrier disruption scoring

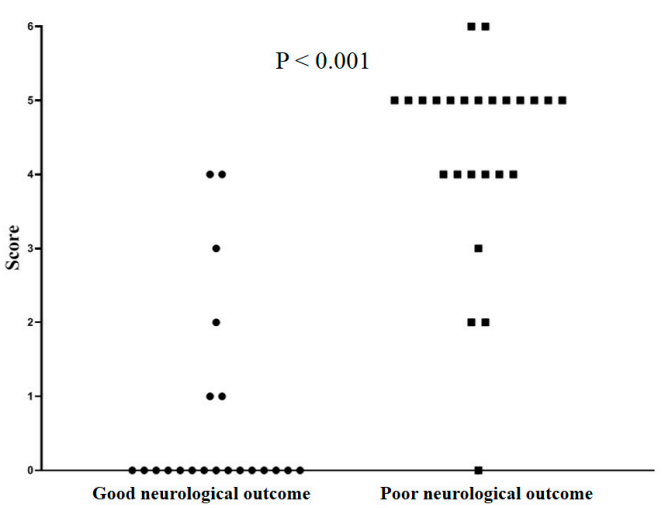

Figure 2. Comparisons between the proportion and the score of blood-brain barrier disruption between the good and the poor neurological outcome groups. (A) In the poor neurological outcome group, the proportions of BBB disruption were significantly higher than those in the good neurological outcome group $(p<0.001)$. (B) In the poor neurological outcome group, the scores of BBB disruption were significantly higher than those in the good neurological outcome group $(p<0.001)$.
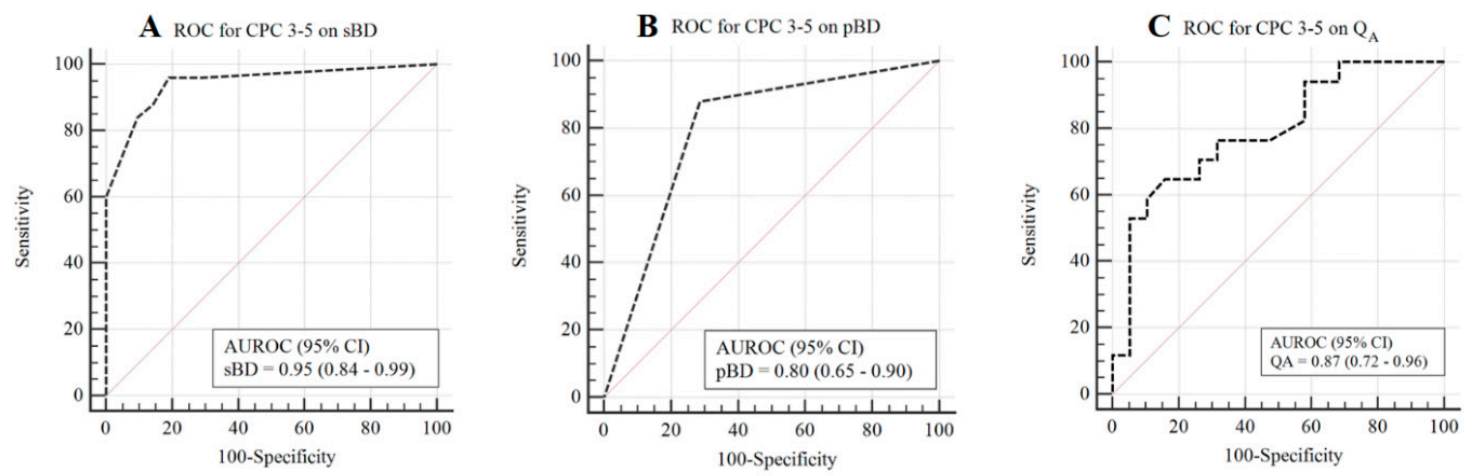

Figure 3. Association of $\mathrm{sBD}, \mathrm{pBD}$ and $\mathrm{Q}_{\mathrm{A}}$ with poor neurological outcomes. (A) Receiver operating characteristic curve for poor neurological outcome on $\mathrm{SBD}$ is shown on the panel. (B) Receiver operating characteristic curve for poor neurological outcome on $\mathrm{pBD}$ is shown on the panel. (C) Receiver operating characteristic curve for poor neurological outcome on $\mathrm{Q}_{\mathrm{A}}$ is shown on the panel.

Table 4. Prognostic performances of sBD, $\mathrm{pBD}$ and $\mathrm{Q}_{\mathrm{A}}$ for neurological outcome.

\begin{tabular}{cccccccc}
\hline Characteristics & AUR (95\% CI) & Cut-off & Sensitivity & Specificity & PPV & NPV & $\begin{array}{c}p \text {-Value for AUC } \\
\text { Comparison }\end{array}$ \\
\hline sBD & $0.95(0.84-0.99)$ & $>1$ & 96.0 & 81.0 & 80.0 & 93.7 & Reference \\
pBD & $0.80(0.65-0.90)$ & Presence & 88.0 & 71.4 & 78.6 & 83.3 & 0.015 \\
$\mathrm{Q}_{\mathrm{A}}$ & $0.87(0.72-0.96)$ & $>0.0133$ & 66,7 & 100.0 & 100.0 & 68.2 & 0.013 \\
\hline
\end{tabular}

AUR, the area under the receiver operating characteristic curve; PPV, positive predictive value; NPV, negative predictive value; $\mathrm{sBD}$, blood-brain barrier disruption score; $\mathrm{pBD}$, presence of blood-brain barrier disruption; $\mathrm{Q}_{\mathrm{A}}$, cerebrospinal fluid-serum albumin quotient.

\subsection{A Comparison of the Relationship between the $Q_{A}$ and $s B D$ and their Ability to Predict Neurological Outcome}

Table 4 shows the relationship between the $\mathrm{Q}_{\mathrm{A}}$ and neurological outcome. In total, $36 \mathrm{Q}_{\mathrm{A}}$ were acquired in 27 patients. The $Q_{A}$ was $0.02(0.009-0.0546)$ in the poor neurological outcome group and $0.0083(0.0053-0.0131)$ in the good neurological outcome group $(p=0.003)$. According to the level of BBB disruption between the good and poor neurological outcome groups, there were $11(57.9 \%)$ vs. $15(88.2 \%)$ patients with more than mild BBB disruption $(p=0.065), 5(26.3 \%)$ vs. $12(70.6 \%)$ patients with more than moderate BBB disruption $(p=0.018)$, and $1(5.3 \%)$ vs. $8(47.1 \%)$ patients with severe 
BBB disruption, respectively, $(p=0.006)$ (Table 5$)$. In addition, the ability to predict poor neurological outcomes in the $\mathrm{SBD}$ was significantly higher than in the $\mathrm{Q}_{\mathrm{A}}(p=0.013)$ (Table 4$)$.

Table 5. Relationship between cerebrospinal fluid/serum albumin quotient and neurological outcome measured in 36 samples.

\begin{tabular}{cccc}
\hline Characteristics & $\begin{array}{c}\text { Good Neurological } \\
\text { Outcome }(\boldsymbol{n = 1 9 )}\end{array}$ & $\begin{array}{c}\text { Poor Neurological } \\
\text { Outcome }(\boldsymbol{n}=\mathbf{1 7})\end{array}$ & $\boldsymbol{p}$-value \\
\hline Total cohort, median (IQR) & $0.0083(0.0053-0.0131)$ & $0.02(0.009-0.0546)$ & 0.003 \\
More than mild BBB disruption, $n(\%)$ & $11(57.9 \%)$ & $15(88.2 \%)$ & 0.065 \\
More than moderate disruption, $n(\%)$ & $5(26.3 \%)$ & $12(70.6 \%)$ & 0.018 \\
Severe BBB disruption, $n(\%)$ & $1(5.3 \%)$ & $8(47.1 \%)$ & 0.006 \\
\hline
\end{tabular}

IQR, interquartile range.

\section{Discussion}

In this retrospective observational study, qualitative (pBD) and quantitative (sBD) analyses of BBB disruption using CE-MRI showed a significant difference between the good and poor neurological outcome groups after 3 months in OHCA survivors treated with TTM. Quantitative analysis predicted prognostic performance better than qualitative analysis. In addition, the sBD showed a significantly higher neurological outcome predictive performance than the $Q_{A}$.

Transient global ischemic brain injury from CA may result in increased permeability and disruption of BBB tight junctions. In particular, leukocytes activated during reperfusion interact with endothelial cells and plug capillaries and interfere with the BBB through the release of neutrophil-derived oxidants and proteolytic enzymes $[15,16]$. Several previous studies involving patients with ischaemic stroke have reported that BBB disruption during cerebral reperfusion may lead to the development of vasogenic oedema and a poor neurological outcome [17-21]. In addition, our previous study also reported that severe $\mathrm{BBB}$ disruption, indicated as a $\mathrm{Q}_{\mathrm{A}} \geq 0.02$, occurred within the first $24 \mathrm{~h}$ after ROSC in a patient group with poor neurological outcomes who had been treated with TTM [9]. However, the $Q_{A}$ has limitations in routine clinical use because CSF is required for the $Q_{A}$.

Under normal physiological conditions, contrast-enhanced FLAIR images cannot detect BBB disruption during reperfusion, because contrast agents do not cross the intact BBB [16]. In a study using postcontrast FLAIR images (termed hyperintense acute perfusion marker, HARM) to characterise early BBB disruption, Steven and Lawrence reported that HARM was found in 47 of 144 patients with ischaemic stroke, and was related to haemorrhagic transformation and poor clinical outcome [17]. In our study, the poor outcome group showed more BBB disruption than the good outcome group ( $88 \%$ vs. $28.6 \%$, respectively). In addition, our study showed that the sBD was superior to the $\mathrm{pBD}$ in predicting neurological outcome. Two explanations are possible for these results. First, an absence of intracranial blood flow due to severe brain oedema was observed in 2 patients, who both showed a poor neurological outcome. These patients had the highest score, i.e., 6 in the sBD, but had negative results in terms of the $\mathrm{pBD}$. Second, a recent retrospective study of 128 patients that investigated the relationship between BBB disruption and neurological complications in patients undergoing endovascular treatment of unruptured intracranial aneurysms reported that an increasing extent of BBB disruption was associated with the development of a neurological event [22]. In this study, BBB disruptions were noted in 2 patients who had an sBP score of 1 point, both of whom had a good neurological outcome. When compared to the cut-off value ( $\mathrm{BBD}>1, \mathrm{pBD}$; presence), patients are expected to have a good neurological outcome with an $\mathrm{sBD}$, but a poor neurological outcome with a positive $\mathrm{pBD}$. 
The $\mathrm{Q}_{\mathrm{A}}$ has been widely accepted as the gold standard for the functional assessment of BBB disruption [12]. However, in this study, CE-MRI was used to determine the presence and degree of BBB disruption. While CE-MRI is a more costly procedure than obtaining a $Q_{A}$, it has the advantage of excellent visualisation and clinical application for assessing BBB disruption and hypoxic ischaemic-reperfusion brain injury [18]. In addition, in this study, the sBD showed better predictability for poor neurological outcome at 3 months after ROSC than the $Q_{A}$. These results may be explained as follows. First, gadobutrol has a molecular weight of 550 Dalton (Da) [22], which is a smaller molecular weight than that of albumin (molecular weight, 66,700 Da) [23]. If BBB disruption occurs, both substances pass through the BBB; however, gadobutrol, which is approximately 100 times smaller in size, is likely to be more permeable and sensitive than albumin. Second, since haemolysis may increase albumin levels, it is also possible that some patients have increased CSF albumin levels without necessarily reflecting brain injury due to haemolysis during the lumbar catheter insertion.

In addition, Son et al.'s recent study showing poor neurological outcome using DWI-MRI conducted before TTM after ROSC reported a high signal intensity in 22 of 33 (66.7\%) patients [8]. One previous study reported that, while DWI is not noticeable in most patients with transient ischemic attack, BBB interruptions may exist. Therefore, HARM can be an additional useful diagnostic tool for assessing patients with acute ischemic stroke [14]. In future, it may be necessary to examine whether combining the sBD and DWI-MRI brain scans provides better predictions concerning neurological outcomes in CA survivors.

This study had several limitations. First, since this is a single-centre retrospective study with a small number of patients, it may affect the selected threshold or other statistical outcome of this work; therefore, a multicentre prospective study is required to enhance the generalisability of the findings. Second, patients were evaluated according to brain CE-MRI and/or CSF and serum albumin levels. However, CE-MRI scans before TTM and lumbar catheter insertion are rare in clinical practice and are very complex to apply; hence, they are generally not used. Third, bias was possible due to the treating physicians being aware of the CSF results or the serum albumin levels. Fourth, CE-MRI scans were performed in all patients, but the inability to obtain CSF albumin levels in some patients may have contributed to bias. In addition, the inability to obtain CSF albumin levels in patients with suspected brain death may have a significant effect on the identification of the prognostic performance of the $Q_{A}$. While there was a small number of patients for whom we could not obtain a $Q_{A}$, given the ratio in terms of the total number of patients, this could have had a considerable effect on our results. Fifth, we used a manual scoring system in this study. However, in future studies involving a larger population, it would be appropriate to use "post-pre subtraction image processing tool" to make more objective and quicker judgments.

\section{Conclusions}

Using CE-MRI, the sBD was shown to be a significantly predictive marker of poor neurological outcome at 3 months in OHCA survivors compared to the $\mathrm{pBD}$ and the $\mathrm{Q}_{\mathrm{A}}$. Multicentre prospective studies are required to determine the generalisability of these results.

Author Contributions: Conceptualization, H.I.K., I.H.L., and J.S.P.; formal analysis, Y.Y.; investigation, J.H.M., W.J.J., Y.C.C. and H.J.A.; methodology, C.K. and B.K.L.; software, J.S.P., D.M.K. and C.K.; supervision, B.K.L.; visualization, C.K.; writing—original draft, H.I.K. and J.S.P.; writing, review and editing, I.H.L., J.S.P., and B.K.L.; funding acquisition, Y.Y. All authors have read and agreed to the published version of the manuscript.

Funding: Y. You received a National Research Foundation of Korea (NRF) grant funded by the Korea government (MSIT) (No. 2019R1F1A1041024).

Conflicts of Interest: The authors declare no conflict of interest. 


\section{Abbreviations}

$\begin{array}{ll}\text { BBB } & \text { blood-brain barrier } \\ \text { CE-MRI } & \text { contrast-enhanced magnetic resonance imaging } \\ \text { OHCA } & \text { out-of-hospital cardiac arrest } \\ \text { pBD } & \text { presence of blood-brain barrier disruption } \\ \text { sBD } & \text { blood-brain barrier disruption score } \\ \text { AUC } & \text { area under the curve } \\ \text { CI } & \text { confidence interval } \\ \text { CA } & \text { cardiac arrest } \\ \text { ROSC } & \text { return of spontaneous circulation } \\ \text { CT } & \text { computed tomography } \\ \text { CPR } & \text { cardiopulmonary resuscitation } \\ \text { CSF } & \text { cerebrospinal fluid } \\ \text { Q } & \text { albumin quotient } \\ \text { TTM } & \text { target temperature management } \\ \text { ECMO } & \text { extracorporeal membrane oxygenation } \\ \text { CPC } & \text { cerebral performance category } \\ \text { FLAIR } & \text { fluid attenuated inversion recovery } \\ \text { ROC } & \text { receiver operating characteristic } \\ \text { AUROC } & \text { area under the ROC } \\ \text { MRI } & \text { magnetic resonance imaging } \\ \text { DWI } & \text { diffusion-weighted image } \\ \text { HARM } & \text { hyperintense acute perfusion marker } \\ & \end{array}$

\section{References}

1. Hayman, E.G.; Patel, A.P.; Kimberly, W.T.; Sheth, K.N.; Simard, J.M. Cerebral Edema after Cardiopulmonary Resuscitation: A Therapeutic Target Following Cardiac Arrest? Neurocrit. Care 2018, 28, 276-287. [CrossRef] [PubMed]

2. Starodub, R.; Abella, B.S.; Grossestreuer, A.V.; Shofer, F.S.; Perman, S.M.; Leary, M.; Gaieski, D.F. Association of serum lactate and survival outcomes in patients undergoing therapeutic hypothermia after cardiac arrest. Resuscitation 2013, 84, 1078-1082. [CrossRef]

3. Xiao, F. Bench to bedside: Brain edema and cerebral resuscitation: The present and future. Acad. Emerg. Med. 2002, 9, 933-946. [CrossRef] [PubMed]

4. Sandroni, C.; Cariou, A.; Cavallaro, F.; Cronberg, T.; Friberg, H.; Hoedemaekers, C.; Horn, J.; Nolan, J.P.; Rossetti, A.O.; Soar, J. Prognostication in comatose survivors of cardiac arrest: An advisory statement from the European Resuscitation Council and the European Society of Intensive Care Medicine. Resuscitation 2014, 85, 1779-1789. [CrossRef]

5. Sandroni, C.; D’Arrigo, S.; Nolan, J.P. Prognostication after cardiac arrest. Crit. Care 2018, 22, 150. [CrossRef] [PubMed]

6. Jeon, C.H.; Park, J.S.; Lee, J.H.; Kim, H.; Kim, S.C.; Park, K.H.; Yi, K.S.; Kim, S.M.; Youn, C.S.; Kim, Y.M.; et al. Comparison of brain computed tomography and diffusion-weighted magnetic resonance imaging to predict early neurologic outcome before target temperature management comatose cardiac arrest survivors. Resuscitation 2017, 118, 21-26. [CrossRef] [PubMed]

7. Park, J.S.; In, Y.N.; You, Y.H.; Min, J.H.; Ahn, H.J.; Yoo, I.S.; Kim, S.W.; Lee, J.W.; Ryu, S.; Jeong, W.J.; et al. Ultra-early neurologic outcome prediction of out-of-hospital cardiac arrest survivors using combined diffusion-weighted imaging findings and quantitative analysis of apparent diffusion coefficient. Resuscitation 2020, 148, 39-48. [CrossRef] [PubMed]

8. Son, S.H.; Lee, I.H.; Park, J.S.; Yoo, I.S.; Kim, S.W.; Lee, J.W.; Ryu, S.; You, Y.; Min, J.H.; Cho, Y.C.; et al. Does Combining Biomarkers and Brain Images Provide Improved Prognostic Predictive Performance for Out-Of-Hospital Cardiac Arrest Survivors before Target Temperature Management? J. Clin. Med. 2020, 9, 744. [CrossRef] 
9. Park, J.S.; You, Y.; Min, J.H.; Yoo, I.; Jeong, W.; Cho, Y.; Ryu, S.; Lee, J.; Kimm, S.W.; Cho, S.U. Study on the timing of severe blood-brain barrier disruption using cerebrospinal fluid-serum albumin quotient in post cardiac arrest patients treated with targeted temperature management. Resuscitation 2019, 135, 118-123. [CrossRef]

10. Takahashi, M.; Macdonald, R.L. Vascular aspects of neuroprotection. Neurol. Res. 2004, 26, 862-869. [CrossRef]

11. Li, J.; Li, C.; Yuan, W.; Wu, J.; Li, J.; Li, Z.; Zhao, Y. Mild hypothermia alleviates brain oedema and blood-brain barrier disruption by attenuating tight junction and adherens junction breakdown in a swine model of cardiopulmonary resuscitation. PLoS ONE 2017, 12, e0174596. [CrossRef] [PubMed]

12. Reiber, H.; Peter, J.B. Cerebrospinal fluid analysis: Disease-related data patterns and evaluation programs. J. Neurol. Sci. 2001, 184, 101-122. [CrossRef]

13. Hansen, M.B.; Tietze, A.; Kalpathy-Cramer, J.; Gerstner, E.R.; Batchelor, T.T.; Ostergaard, L.; Mouridsen, K. Reliable estimation of microvascular flow patterns in patients with disrupted blood-brain barrier using dynamic susceptibility contrast MRI. J. Magn. Reson. Imaging 2017, 46, 537-549. [CrossRef] [PubMed]

14. You, Y.; Park, J.S.; Min, J.; Yoo, I.; Ahn, H.J.; Cho, Y.; Ryu, S.; Lee, J.; Kim, S.; Cho, S.; et al. The usefulness of neuron-specific enolase in cerebrospinal fluid to predict neurological prognosis in cardiac arrest survivors who underwent target temperature management: A prospective observational study. Resuscitation 2019, 145, 185-191. [CrossRef] [PubMed]

15. Kuroda, S.; Siesjö, B.K. Reperfusion damage following focal ischemia: Pathophysiology and therapeutic windows. Clin. Neurosci. 1997, 4, 199-212. [PubMed]

16. Pan, J.; Konstas, A.A.; Bateman, B.; Ortolano, G.A.; Pile-Spellman, J. Reperfusion injury following cerebral ischemia: Pathophysiology, MR imaging, and potential therapies. Neuroradiology 2007, 49, 93-102. [CrossRef]

17. Warach, S.; Latour, L.L. Evidence of reperfusion injury, exacerbated by thrombolytic therapy, in human focal brain ischemia using a novel imaging marker of early blood-brain barrier disruption. Stroke 2004, 35 (Suppl. 1), 2659-2661. [CrossRef] [PubMed]

18. Li, Y.; Kim, J.; Simpson, D.; Aagaard-Kienitz, B.; Niemann, D.; Esene, I.N.; Ahmed, A. Difference in imaging biomarkers between transient and permanent neurological deficits after endovascular treatment of cerebrovascular aneurysms. J. Neurosurg. 2020, 1, 1-10.

19. Förster, A.; Wenz, H.; Groden, C. Hyperintense Acute Reperfusion Marker on FLAIR in a Patient with Transient Ischemic Attack. Case Rep. Radiol. 2016, 2016, 9829823. [CrossRef]

20. Ogami, R.; Nakahara, T.; Hamasaki, O.; Araki, H.; Kurisu, K. Cerebrospinal fluid enhancement on fluid attenuated inversion recovery images after carotid artery stenting with neuroprotective balloon occlusions: Hemodynamic instability and blood-brain barrier disruption. Cardiovasc. Interv. Radiol. 2011, 34, $936-941$. [CrossRef] [PubMed]

21. Bang, O.Y.; Saver, J.L.; Alger, J.R.; Shah, S.H.; Buck, B.H.; Starkman, S.; Ovbiagele, B.; Liebeskind, D.S. UCLA MRI Permeability Investigators. Patterns and predictors of blood-brain barrier permeability derangements in acute ischemic stroke. Stroke 2009, 40, 454-461. [CrossRef] [PubMed]

22. Eide, P.K.; Ringstad, G. MRI with intrathecal MRI gadolinium contrast medium administration: A possible method to assess glymphatic function in human brain. Acta Radiol. Open 2015, 4, 2058460115609635. [CrossRef] [PubMed]

23. Lee, P.; Wu, X. Review: Modifications of human serum albumin and their binding effect. Curr. Pharm. Des. 2015, 21, 1862-1865. [CrossRef] [PubMed]

(C) 2020 by the authors. Licensee MDPI, Basel, Switzerland. This article is an open access article distributed under the terms and conditions of the Creative Commons Attribution (CC BY) license (http://creativecommons.org/licenses/by/4.0/). 